\title{
Effect of mediated interactions on a Hubbard chain in mixed-dimensional fermionic cold atoms
}

\author{
Junichi Okamoto $\odot,{ }^{1}$ Wen-Min Huang, ${ }^{2,}{ }^{*}$ Kyle Irwin, ${ }^{3}$ David K. Campbell $\odot,{ }^{4}$ and Shan-Wen Tsai ${ }^{3}$ \\ ${ }^{1}$ Physikalisches Institut, Albert-Ludwigs-Universität Freiburg, Hermann-Herder-Straße 3, 79104 Freiburg, Germany \\ ${ }^{2}$ Department of Physics, National Chung-Hsing University, Taichung 40227, Taiwan \\ ${ }^{3}$ Department of Physics and Astronomy, University of California, Riverside, California 92521, USA \\ ${ }^{4}$ Department of Physics, Boston University, Boston, Massachusetts 02215, USA
}

(Received 28 April 2020; accepted 24 June 2020; published 10 July 2020)

\begin{abstract}
Cold atom experiments can now realize mixtures where different components move in different spatial dimensions. We investigate a fermion mixture where one species is constrained to move along a one-dimensional lattice embedded in a two-dimensional lattice populated by another species of fermions, and where all bare interactions are contact interactions. By focusing on the one-dimensional fermions, we map this problem onto a model of fermions with nonlocal interactions on a chain. The effective interaction is mediated by the two-dimensional fermions and is both attractive and retarded, the form of which can be varied by changing the density of the two-dimensional fermions. By using the functional renormalization group in the weak-coupling limit and ignoring the retardation effect, we show that the one-dimensional fermions can be controlled to be in various density-wave, or spin-singlet or spin-triplet superconducting phases.
\end{abstract}

DOI: 10.1103/PhysRevResearch.2.033054

\section{INTRODUCTION}

Cold atom systems have proven to be an invaluable tool in studying a wide range of quantum many-body phenomena. Even a single species of atoms can exhibit various quantum states such as Bose-Einstein condensates (BECs) [1,2], Mott insulators [3], or unitary Fermi gases [4-7]. The complexity of cold atomic systems can be greatly enhanced by mixing different atoms. For example, a degenerate Fermi gas immersed in a BEC can be realized with isotopes of $\mathrm{Li}$ atoms [8-10] or with different atomic species [11,12]. A two-species Fermi gas was first realized with $\mathrm{Li}$ and $\mathrm{K}$ atoms [13], and has been studied extensively [14-16]. The controllability of interspecies interactions permits these ultracold atomic mixtures to become valuable platforms to study novel many-body phenomena such as an impurity problem [17,18], polaron formation [19,20], or lattice gauge theories [21,22].

The development of experimental techniques to create species-specific lattice geometries has further extended the possibilities of the mixed atomic gases [23,24]. For example, in a recent experimental work, a bosonic mixture of ${ }^{41} \mathrm{~K}$ and ${ }^{87} \mathrm{Rb}$ atoms is confined in two dimensions and in three dimensions, respectively [25]. In light of this experimental success, several other experimental works [26,27] have been conducted to create Fermi-Bose mixtures. We also note that a ${ }^{3} \mathrm{He}-{ }^{4} \mathrm{He}$ solution provides effectively mixed-dimensional Fermi gases [28]. Theoretical works on mixed-dimensional

\footnotetext{
*wenmin@phys.nchu.edu.tw

Published by the American Physical Society under the terms of the Creative Commons Attribution 4.0 International license. Further distribution of this work must maintain attribution to the author(s) and the published article's title, journal citation, and DOI.
}

systems predict phenomena such as Efimov effects [29,30], mediated pairing [31-35], or a topological superfluid [36,37].

Motivated by this progress, we propose in the present paper to investigate a Fermi system composed of $c$-type and $f$ type species confined to one dimension and two dimensions, respectively (Fig. 1). We assume a contact interaction in the one-dimensional (1D) system while ignoring the interaction between the two-dimensional (2D) particles. Through the interspecies contact interaction, the $f$ particles induce a mediated interaction of the Ruderman-Kittel-Kasuya-Yosida type [38-40] among the $c$ particles. The momentum structure of the effective interaction is determined by the filling of the $f$ particles and thus can be used as a tuning knob to manipulate the quantum phases in the $1 \mathrm{D}$ system.

In the weak-coupling limit, we investigate the ground state phase diagram by a functional renormalization group (fRG) [41-47]. This treatment enables us to go beyond the standard $g$-ology and bosonization methods [48] by including the curvature of the band dispersion and the momentum dependence of the coupling constants. We identify the dominant instability by looking at the flow of the coupling constants and show that the resulting phase diagram includes charge-/bond-/spin-density waves and spin-singlet/-triplet superconductivity. We find that formally irrelevant terms can play an important role in determining the dominant pairing phase, and also lead to a dominant bond-wave order instability among the many competing ones in a small region of the phase space. When the filling of the $c$ particles is away from half filling, the density-wave instabilities are replaced by the superconducting instabilities. This demonstrates a possible means of manipulating exotic quantum phases such as bonddensity waves or spin-triplet pairing in a mixed-dimensional setup.

The remainder of our paper is organized as follows. Section II introduces the model that we consider and discusses the 


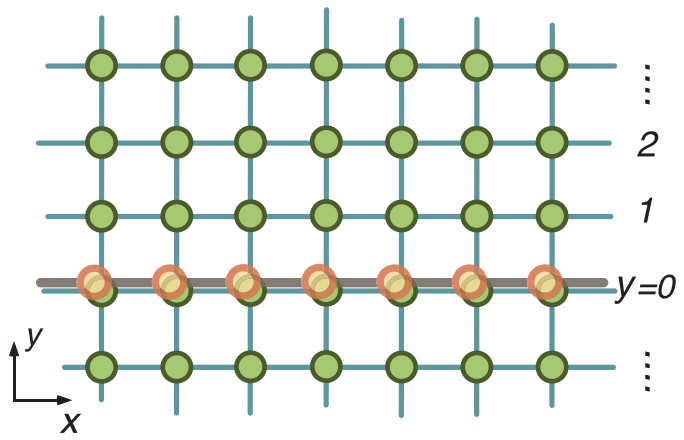

FIG. 1. Schematic of the model that we consider. The onedimensional optical lattice is in contact with the two-dimensional lattice at $y=0$.

mediated interactions in the 1D system. In Sec. III, we briefly summarize the functional renormalization group method as applied to our model, and explain how we determine the phase diagram. Section IV shows the flows of the coupling constants and the phase diagrams. Section V presents our conclusions.

\section{MODEL}

The 1D system that we consider is given by

$$
\begin{aligned}
H_{c}= & -t_{c} \sum_{\left\langle\left\langle x^{\prime}\right\rangle, \sigma\right.}\left(c_{x, \sigma}^{\dagger} c_{x^{\prime}, \sigma}+\text { H.c. }\right) \\
& +U_{c} \sum_{x} n_{x, \uparrow}^{c} n_{x, \downarrow}^{c}-\mu_{c} \sum_{x, \sigma} n_{x \sigma}^{c},
\end{aligned}
$$

where $c_{x \sigma}^{(\dagger)}$ is the annihilation (creation) operator of a $c$ fermion with spin $\sigma$ at site $x$, and $t_{c}$ is the hopping between nearestneighbor sites $\left\langle x, x^{\prime}\right\rangle$ on a regular lattice. $U_{c}$ is the on-site Hubbard interaction between particle densities $n_{x \sigma}^{c}=c_{x \sigma}^{\dagger} c_{x \sigma}$, and $\mu_{c}$ is the chemical potential. Similarly, the 2D system of the noninteracting $f$ fermions is given by

$$
H_{f}=-t_{f} \sum_{\left\langle\boldsymbol{r}, \boldsymbol{r}^{\prime}\right\rangle, \sigma} f_{\boldsymbol{r} \sigma}^{\dagger} f_{\boldsymbol{r}^{\prime} \sigma}-\mu_{f} \sum_{\boldsymbol{r}, \sigma} n_{\boldsymbol{r}, \sigma}^{f},
$$

where $f_{r \sigma}^{(\dagger)}$ is the annihilation (creation) operator of a $f$ fermion with spin $\sigma$ at site $\boldsymbol{r}=(x, y)$, and $t_{f}$ is the hopping between nearest-neighbor sites $\left\langle\boldsymbol{r}, \boldsymbol{r}^{\prime}\right\rangle$ on a square lattice. The two species interact at $y=0$ with spin-independent densitydensity interaction $U_{c f}$,

$$
H_{c f}=U_{c f} \sum_{x, \sigma, \sigma^{\prime}} n_{x \sigma}^{c} n_{x, y=0, \sigma^{\prime}}^{f} .
$$

We consider $N_{0}$ and $N_{0}^{2}$ sites for $c$ and $f$ particles with a periodic boundary condition with a lattice constant $a=1$. The Fourier transforms are given by

$$
\begin{gathered}
c_{x \sigma}=\frac{1}{\sqrt{N_{0}}} \sum_{k \in \mathrm{BZ}} e^{i k x} c_{k \sigma}, \\
f_{\boldsymbol{r} \sigma}=\frac{1}{\sqrt{N_{0}^{2}}} \sum_{\boldsymbol{p} \in \mathrm{BZ}} e^{i \boldsymbol{p} \cdot \boldsymbol{r}} f_{\boldsymbol{p} \sigma},
\end{gathered}
$$

which give rise to the $1 \mathrm{D}$ and $2 \mathrm{D}$ dispersion relations $\xi_{k}^{c}=$ $-2 t_{c} \cos (k)-\mu_{c}$ and $\xi_{p}^{f}=-2 t_{f}\left[\cos \left(p_{x}\right)+\cos \left(p_{y}\right)\right]-\mu_{f}$.
Next, we construct a path integral partition function over Grassmann fields [8], and seek an effective 1D action. The total action of the $1 \mathrm{D}$ and $2 \mathrm{D}$ fermions with imaginary time $\tau$ and inverse temperature $\beta$ is given by

$$
\begin{aligned}
S= & \int_{0}^{\beta} d \tau\left[\sum_{k, \sigma} c_{k \sigma}^{\dagger}(\tau) \partial_{\tau} c_{k \sigma}(\tau)+H_{c}(\tau)\right. \\
& \left.+\sum_{p, \sigma} f_{p \sigma}^{\dagger}(\tau) \partial_{\tau} f_{p \sigma}(\tau)+H_{f}(\tau)+H_{c f}(\tau)\right] .
\end{aligned}
$$

With the Fourier series in Matsubara frequencies $\omega_{n}=$ $\pi(2 n+1) / \beta(n \in \mathbb{Z})$ as

$$
f_{p \sigma}(\tau)=\frac{1}{\sqrt{\beta}} \sum_{n} e^{-i \omega_{n} \tau} f_{n \boldsymbol{p} \sigma},
$$

we can cast the quadratic action of the $2 \mathrm{D}$ fermions into a matrix form,

$$
S_{2 \mathrm{D}}=\sum_{n, n^{\prime}, \boldsymbol{p}, \boldsymbol{p}^{\prime}, \sigma} f_{n \boldsymbol{p} \sigma}^{\dagger}\left[-G_{0}^{-1}+M\right]_{(n \boldsymbol{p}) ;\left(n^{\prime} \boldsymbol{p}^{\prime}\right)} f_{n^{\prime} \boldsymbol{p}^{\prime} \sigma},
$$

with a Green's function matrix $G_{0}$ and an interaction $\operatorname{part} M$,

$$
\begin{aligned}
{\left[G_{0}\right]_{(n \boldsymbol{p}) ;\left(n^{\prime} \boldsymbol{p}^{\prime}\right)} } & =\frac{1}{i \omega_{n}-\xi_{f}(\boldsymbol{p})} \delta_{n n^{\prime}} \delta_{\boldsymbol{p} \boldsymbol{p}^{\prime}}, \\
{[M]_{(n \boldsymbol{p}) ;\left(n^{\prime} \boldsymbol{p}^{\prime}\right)} } & =\frac{U_{c f}}{N_{0}^{3} \beta} \sum_{m, k, \sigma} c_{m k \sigma}^{\dagger} c_{n-n^{\prime}+m, p_{x}-p_{x}^{\prime}+k, \sigma} .
\end{aligned}
$$

After integrating out the 2D fermions, the effective action for the $1 \mathrm{D}$ fermions becomes

$$
\begin{aligned}
S_{\text {eff }} & =S_{c}-2 \operatorname{Tr} \ln \left[-G_{0}^{-1}+M\right] \\
& =S_{c}-2 \operatorname{Tr} \ln \left[-G_{0}^{-1}\left(1-G_{0} M\right)\right] \\
& =S_{c}+2 \sum_{n=1}^{\infty} \frac{\operatorname{Tr}\left[\left(G_{0} M\right)^{n}\right]}{n}+\text { const },
\end{aligned}
$$

where the factor of 2 accounts for the spin of the $f$ particles, taken here to be spin-1/2. The first-order term in the expansion gives the correction to the chemical potential of the 1D fermions, which we assume to be included in $\mu_{c}$ in the following. Summation over $\omega_{n}$ in the second-order term generates the effective interaction,

$$
\begin{aligned}
\operatorname{Tr}\left[\left(G_{0} M\right)^{2}\right] & \\
= & \frac{U_{c f}^{2}}{N_{0}^{6} \beta} \sum_{\tilde{l}, \boldsymbol{p}, \boldsymbol{p}^{\prime}}\left[\frac{n_{\mathrm{F}}\left(\xi_{\boldsymbol{p}}^{f}\right)-n_{\mathrm{F}}\left(\xi_{\boldsymbol{p}+\boldsymbol{p}^{\prime}}^{f}\right)}{i \tilde{\omega}_{l}+\xi_{\boldsymbol{p}}^{f}-\xi_{\boldsymbol{p}+\boldsymbol{p}^{\prime}}^{f}}\right. \\
& \left.\times \sum_{m, m^{\prime}, k, k^{\prime}, \sigma, \sigma^{\prime}} c_{m k \sigma}^{\dagger} c_{m-\tilde{l},-p_{x}^{\prime}+k, \sigma} c_{m^{\prime} k^{\prime} \sigma^{\prime}}^{\dagger} c_{m^{\prime}+\tilde{l}, p_{x}^{\prime}+k^{\prime}, \sigma^{\prime}}\right],
\end{aligned}
$$

where $\tilde{\omega}_{l}=\omega_{n^{\prime}}-\omega_{n}$ is a bosonic Matsubara frequency, and $n_{\mathrm{F}}(\xi)$ is the Fermi distribution function. If we consider $t_{f} \gg$ $t_{c} \gg 1 / \beta$, we can ignore the retardation effects, and only the $\tilde{\omega}_{l}=0$ component is important. In this limit, we can write a mediated interaction for the $c$ fermions, Eq. (10), in 


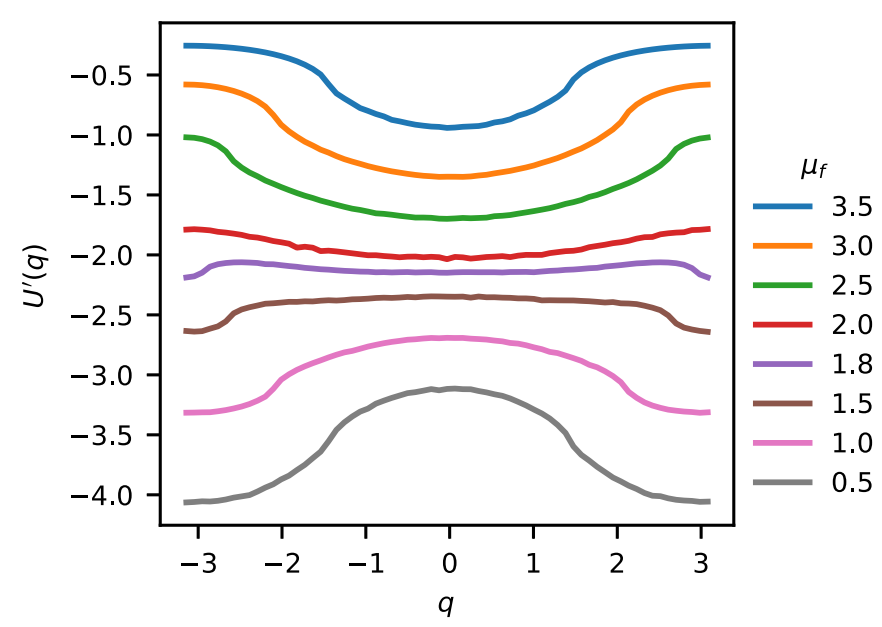

FIG. 2. Mediated interaction $U^{\prime}(q)$ in Eq. (11) in units of $U_{c f}^{2} / t_{f}$. As the chemical potential of the $f$ particle increases, the dominant component shifts from $U^{\prime}(\pi)$ to $U^{\prime}(0)$.

momentum space as

$$
\begin{aligned}
H_{\mathrm{med}} & =\frac{1}{2 N_{0}} \sum_{k, k^{\prime}, q, \sigma, \sigma^{\prime}} U^{\prime}(q) c_{k \sigma}^{\dagger} c_{k^{\prime} \sigma^{\prime}}^{\dagger} c_{k^{\prime}+q \sigma^{\prime}} c_{k-q \sigma}, \\
U^{\prime}(q) & =2 U_{c f}^{2} \int_{-\pi}^{\pi} \frac{d \boldsymbol{p} d p_{y}^{\prime}}{(2 \pi)^{3}} \frac{n_{F}\left(\xi_{p}^{f}\right)-n_{F}\left(\xi_{p+\left(q, p_{y}^{\prime}\right)}^{f}\right)}{\xi_{p}^{f}-\xi_{p+\left(q, p_{y}^{\prime}\right)}^{f}},
\end{aligned}
$$

where the strength of the mediated interaction scales as $U_{c f}^{2} / t_{f}$.

We plot the induced interaction in Fig. 2 for various fillings of the $2 \mathrm{D}$ system. In what follows, the mediated interaction for momentum transfers consistent with the marginal scattering processes [4,9] plays the key role in the development of various quantum phases of the system. At $\mu_{f}=0,\left|U^{\prime}(\pi)\right|>$ $\left|U^{\prime}(0)\right|$. However, as we increase $\mu_{f}, U^{\prime}(0) / U^{\prime}(\pi)$ becomes larger, and around $\mu_{f} \approx 1.8 t_{f}$, the magnitudes of $U^{\prime}(0)$ and $U^{\prime}(\pi)$ switch; this signals the appearance of different leadingorder instabilities, as will be shown below. With the original interaction $U_{c}$ included, the 1D system now has the total interaction $V\left(k_{1}, k_{2}, k_{3}\right)$ as

$$
V\left(k_{1}, k_{2}, k_{3}\right)=U_{c}+U^{\prime}\left(k_{3}-k_{2}\right),
$$

where the fourth momentum (not explicitly written above) is automatically determined by the momentum conservation. It follows that by varying the interactions $U_{c}$ and $U_{c f}$ and the 2D filling $\mu_{f}$, we can control $U^{\prime}(0)$ and $U^{\prime}(\pi)$ to any negative value, while the overall strength of the interaction must be small enough for the perturbative scheme to be valid.

\section{METHOD}

Based on the effective interaction, phase diagrams are obtained by a fRG scheme. Here, we briefly outline the standard $N$-patch scheme [41,43-45,47], which we employ in this work. We divide the Brillouin zone into $N_{\text {patch }}$ patches as shown in Fig. 3, where the patch momenta $\left\{\bar{k}_{n}\right\}$ are equally spaced. The interaction is now approximated as $V\left(k_{1}, k_{2}, k_{3}\right) \rightarrow V_{n_{1} n_{2} n_{3}}$, where $n_{i}$ is the patch that $k_{i}$ belongs to. Naively, the total number of coupling constants is $N_{\text {patch }}^{3}=$

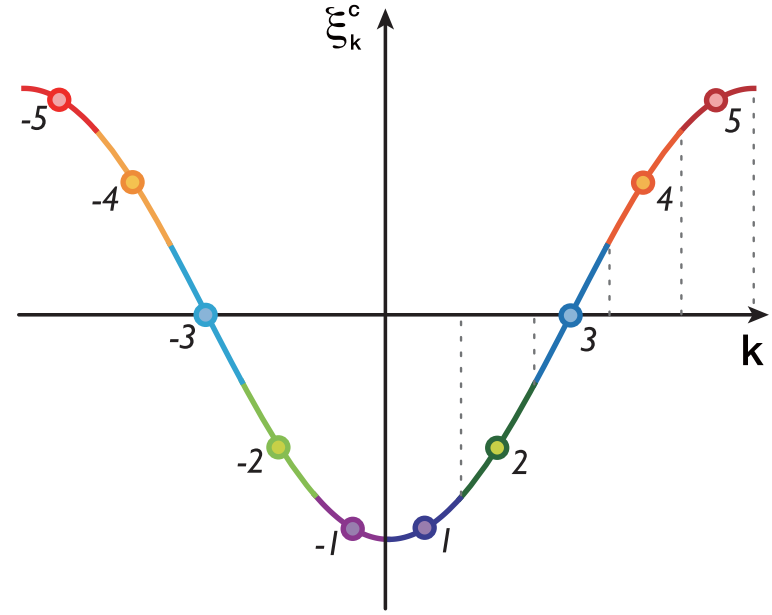

FIG. 3. Patching scheme for the functional renormalization group.

27000 . However, we can reduce this number by using the symmetry of the Hamiltonian to 3735 . The RG equation is obtained after integrating out the high-energy degrees of freedom around the ultraviolet cutoff $\Lambda$. By parametrizing the cutoff as $\Lambda(l)=\Lambda_{0} e^{-l}$ with the initial value of the cutoff $\Lambda_{0}$, the coupling constants at lower energies are obtained by integrating the RG equations [45,47],

$$
\begin{aligned}
\frac{\partial V_{n_{1} n_{2} n_{3}}}{\partial l}= & -\sum_{n} \dot{\Pi}^{-}\left(n, q_{\mathrm{pp}}\right)\left(V_{n_{1} n_{2} n} V_{n_{4} n_{3} n}+V_{n_{2} n_{1} n} V_{n_{3} n_{4} n}\right) \\
& +\sum_{n} \dot{\Pi}^{+}\left(n, q_{\mathrm{fs}}\right)\left(2 V_{n n_{4} n_{1}} V_{n n_{2} n_{3}}-V_{n_{4} n n_{1}} V_{n n_{2} n_{3}}\right. \\
& \left.-V_{n n_{4} n_{1}} V_{n_{2} n n_{3}}\right) \\
& +\sum_{n} \dot{\Pi}^{+}\left(n,-q_{\mathrm{fs}}\right)\left(2 V_{n n_{1} n_{4}} V_{n n_{3} n_{2}}-V_{n_{1} n n_{4}} V_{n n_{3} n_{2}}\right. \\
& \left.-V_{n n_{1} n_{4}} V_{n_{3} n n_{2}}\right) \\
& -\sum_{n} \dot{\Pi}^{+}\left(n, q_{\mathrm{ex}}\right) V_{n_{3} n n_{1}} V_{n_{2} n n_{4}} \\
& -\sum_{n} \dot{\Pi}^{+}\left(n,-q_{\mathrm{ex}}\right) V_{n_{1} n n_{3}} V_{n_{4} n n_{2}}
\end{aligned}
$$

where $q_{\mathrm{pp}}=\bar{k}_{n_{1}}+\bar{k}_{n_{2}}, q_{\mathrm{fs}}=\bar{k}_{n_{3}}-\bar{k}_{n_{2}}$, and $q_{\mathrm{ex}}=\bar{k}_{n_{1}}-\bar{k}_{n_{3}}$. $\dot{\Pi}^{ \pm}(n, q)$ is a differential of a bubble integral over frequency $\omega$ and momentum $k$ inside the $n$th patch,

$$
\dot{\Pi}^{ \pm}(n, q)= \pm \Lambda \int_{\omega} \int_{k \in n} \dot{G}(\omega, k) G[ \pm \omega, \pm(k-q)]
$$

with $G(\omega, k)=\Theta\left(\left|\xi_{k}^{c}\right|-\Lambda\right) /\left(i \omega-\xi_{k}^{c}\right)$. Here, the free propagator is used, since we ignore the self-energy correction along the RG flows.

A RG flow is started from an ultraviolet cutoff $\Lambda_{0}=2 t_{c}$ and integrated until one of the coupling constants becomes $\sim 20 t_{c}$ or $\Lambda=10^{-6} t$. The former indicates an ordering instability, while the latter indicates no instability, i.e., the Luttinger liquid fixed point. To determine the dominant instability, we extract the coupling constants at the Fermi level 
(a) $\mu_{c}=0$
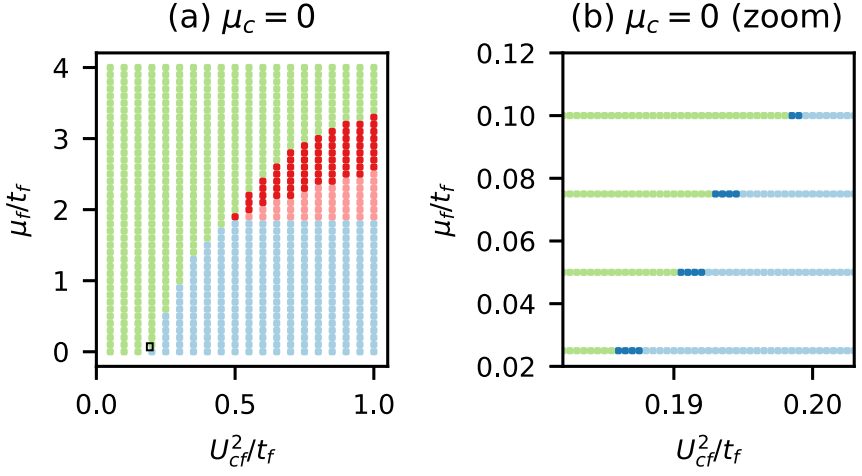

CDW

BCDW

SDW

SS

TS

FIG. 4. Phase diagrams for $\mu_{c}=0$ (half filling). The rectangular region in (a) is enlarged in (b) to zoom into the CDW-SDW boundary, showing the narrow BCDW phase.

as

$$
\begin{aligned}
& g_{1}=V(L, R, L), \\
& g_{2}=V(L, R, R), \\
& g_{3}=V(L, L, R),
\end{aligned}
$$

where $L, R$ denote the left and right patches that the Fermi momentum is contained. This connects our treatment to the standard $g$-ology analysis, which usually assumes linear dispersion around the Fermi energy. In our analysis, the flows of these coupling constants are affected by the curvature of the band dispersion and by other marginal and irrelevant coupling constants away from the Fermi energy. As we discuss in the next section, these corrections are important to capture the emergence of a bond-order phase and subtle competition between pairing phases. We consider charge-density waves (CDWs), spin-density waves (SDWs), spin-singlet superconductivity (SS), and spin-triplet superconductivity (TS). When the system is at half filling, bond charge-/spin-density waves (BCDWs/BSDWs) are also possible. The ordering tendencies of these phases are measured by [49]

$$
\begin{aligned}
g_{\mathrm{CDW} / \mathrm{BCDW}} & =-2 g_{1}+g_{2}\left(\mp g_{3}\right), \\
g_{\mathrm{SDW} / \mathrm{BSDW}} & =g_{2}\left( \pm g_{3}\right), \\
g_{\mathrm{SS} / \mathrm{TS}} & =\mp g_{1}-g_{2},
\end{aligned}
$$

where the $g_{3}$ term is omitted for non-half-filling conditions. We identify the leading instability by the most diverging coupling constant in Eq. (16).

\section{RESULTS}

\section{Phase diagrams}

We start from the phase diagrams for half filling, $\mu_{c}=0$, in Fig. 4(a) at $U_{c}=t_{c}$. When the interspecies interaction $U_{c f}$ is weak or the $2 \mathrm{D}$ filling is very high, the total interaction is dominated by $U_{c}$, which leads to the SDW instability. As $U_{c f}$ increases, the mediated attractive interaction becomes stronger. When the $2 \mathrm{D}$ fermions are half filled $\mu_{f}=0$ or close to it, the mediated interaction is dominated by the $q=\pi$ component, with $\left|U^{\prime}(q=\pi)\right|>\left|U^{\prime}(q=0)\right|$, as shown
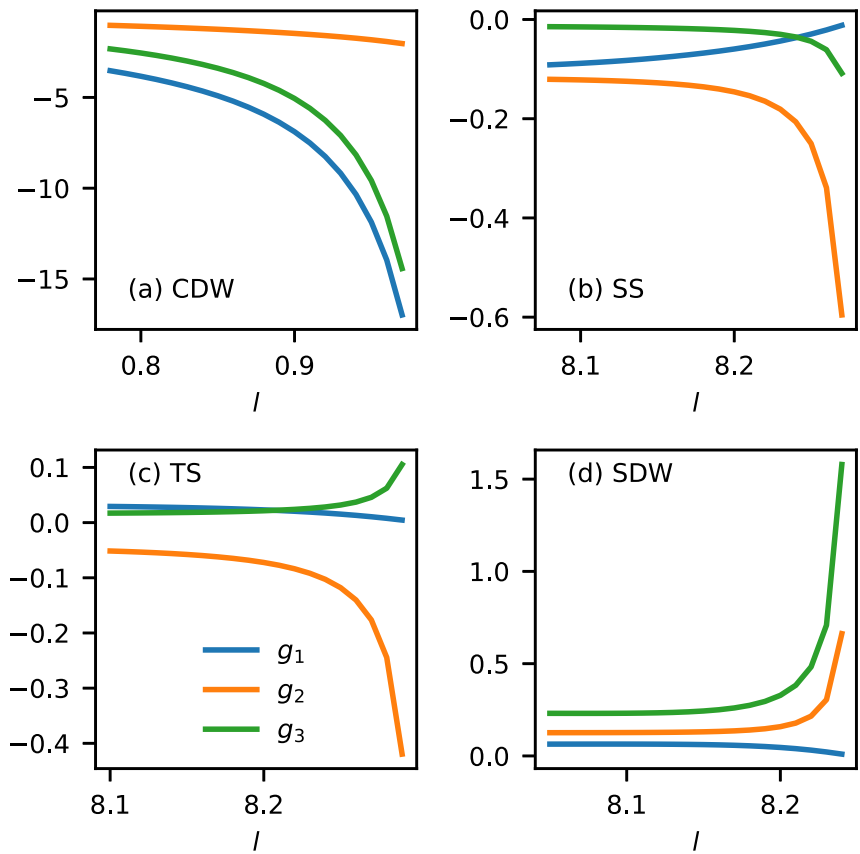

FIG. 5. Flows of the coupling constants $g_{1,2,3}(l)$ for CDW (upper left), SS (upper right), TS (lower left), and SDW (lower right) regions at $U_{c f}^{2} / t_{f}=0.8$. The values of $\mu_{f} / t_{f}$ are $0.0,2.1,2.7$, and 3.7, respectively.

in Fig. 2, and CDW order becomes dominant. However, when the 2D filling deviates from half filling significantly $\mu_{f} \gtrsim 1.8 t_{f}, U^{\prime}(q=0)$ becomes the largest component in the mediated attractive interaction (see Fig. 2), which leads to spin-singlet pairing (SS). At $\mu_{f} \approx 1.8 t_{f}$, the mediated interaction is independent of momentum $q$, and thus gives attractive on-site interactions in real space. Hence, at $U_{c f}^{2} / t_{f} \approx 0.55$, the original repulsive on-site Hubbard $U_{c}$ and the mediated contribution cancel, leading to a quadruple point where SDW, CDW, SS, and TS meet (Fig. 4). At this point, the system is noninteracting and behaves as a Luttinger liquid. A further increase of the 2D filling leads to spin-triplet pairing (TS) and then eventually the mediated interaction becomes too weak. We consider the TS region to be reminiscent of the $d$-wave pairing in a two-leg ladder with mediated interactions [32].

At half filling, it is known that the extended Hubbard model exhibits BCDW at the boundary between CDW and SDW at a weak-coupling regime, i.e., $g_{1}=g_{3} \simeq 0$ at the bare level [43,50-54]. In Fig. 4(b), we plot the phase diagram near the CDW-SDW boundary to demonstrate the existence of the BCDW. The BCDW appears when $g_{1} \simeq g_{3} \simeq 0$ at the bare level as in the extended Hubbard model. While the $\mathrm{BCDW}$ region is rather narrow, our model correctly captures the known BCDW phase at the CDW-SDW boundary because of the radial patch scheme we employed for our fRG calculations. This region can be enlarged by increasing the original on-site interaction $U_{c}$, and the width of the BCDW is largest at $\mu_{f}=0$, and becomes smaller as increasing $\mu_{f}$. This indicates that the BCDW can be enhanced by having larger values of $U^{\prime}(\pi)$.

In order to further understand the origin of each region, we plot the flows of the coupling constants in Fig. 5. In the CDW 
(a) $\mu_{c}=0.5 t_{c}$

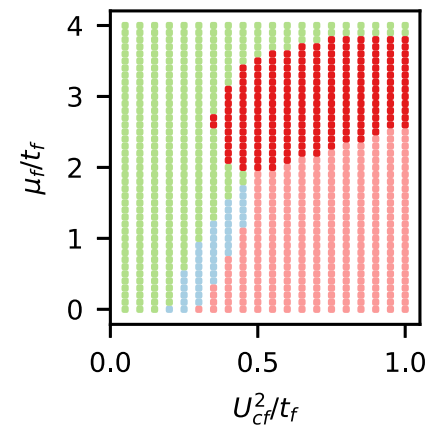

(b) $\mu_{c}=1.5 t_{c}$

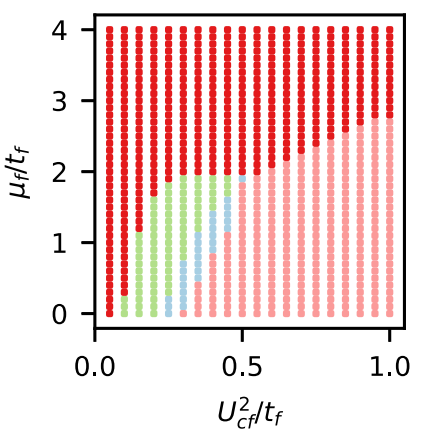

CDW SDW

- TS

FIG. 6. Phase diagrams for (a) $\mu_{c}=0.5 t_{c}$ and (b) $\mu_{c}=1.5 t_{c}$.

region, the large negative value of $g_{1}$ dominates. For $\mu_{f} \simeq 0$, the subdominant coupling is negative $g_{3}$ as in Fig. 5(a), while as we approach the SS region, $g_{2}$ becomes subdominant. The growth of $g_{2}$ switches the dominant instability from the CDW to the SS. In the SS region [Fig. 5(b)], $g_{2}$ goes to $-\infty$, while $g_{1}$ and $g_{3}$ remain very small. Due to the small negative $g_{1}$, the SS instability is more dominant than the TS. In the TS region [Fig. 5(c)], $g_{2}$ still diverges to $-\infty$, while $g_{3}$ also grows, which signals the closeness to SDW. Here, due to the small positive $g_{1}$, the TS instability is more dominant than the SS. These indicate that the difference between the SS and TS instabilities is very subtle and determined by the marginally irrelevant $g_{1}$ coupling. The same behaviors are also seen in the extended Hubbard model in Ref. [49]. Finally, in the SDW region [Fig. 5(d)], both $g_{2}$ and $g_{3}$ diverge to $+\infty$ while $g_{1}$ is marginally irrelevant. Here, both the SDW and BCDW instabilities are possible, while the small positive $g_{1}$ makes the SDW more stable.

Lastly, we show phase diagrams for $\mu_{c} \neq 0$ in Fig. 6. In general, $g_{2}$ becomes the most dominant term, and thus both SS and TS regions are enhanced. Compared to the half-filling case, at $\mu_{c}=0.5 t_{c}$, the CDW region is greatly reduced, due to the absence of the umklapp term $g_{3}$, while the SDW region mostly remains. However, as we increase the filling to $\mu_{c}=$ $1.5 t_{c}$, the SDW instability is replaced by the TS, and the density waves only exist for relatively small $\mu_{f}$ and $U_{c f}$.

\section{CONCLUSIONS}

In this paper, we have studied the effect of mediated interactions on a Hubbard chain in contact with a two-dimensional noninteracting Fermi gas. After integrating out the fast noninteracting two-dimensional particles, we derive an effective mediated interaction among the one-dimensional fermions, whose momentum-dependent structure can be controlled by the filling of the mediating particles. With the mediated interaction and the original Hubbard interaction, we have obtained ground state phase diagrams of the one-dimensional fermions by the functional renormalization group. We find that the system can exhibit charge-/spin-/bond-density waves and spin-singlet/-triplet superconducting instabilities by controlling the filling of the two-dimensional particles and the interspecies interaction. The effects of trapping potentials, temperatures, and interactions among the $f$ particles are interesting open problems.

\section{ACKNOWLEDGMENTS}

J.O. acknowledges support by the Georg H. Endress Foundation and by the state of Baden-Württemberg through bwHPC. S.-W.T. acknowledges support from NSF under Grant No. PHY-1839153. W.-M.H. acknowledges support from the Ministry of Science and Technology, Taiwan through Grant No. MOST 107-2112-M-005-008-MY3.
[1] M. H. Anderson, J. R. Ensher, M. R. Matthews, C. E. Wieman, and E. A. Cornell, Observation of Bose-Einstein condensation in a dilute atomic vapor, Science 269, 198 (1995).

[2] K. B. Davis, M.-O. Mewes, M. R. Andrews, N. J. van Druten, D. S. Durfee, D. M. Kurn, and W. Ketterle, Bose-Einstein Condensation in a Gas of Sodium Atoms, Phys. Rev. Lett. 75, 3969 (1995).

[3] M. Greiner, O. Mandel, and T. Esslinger, Quantum phase transition from a superfluid to a Mott insulator in a gas of ultracold atoms, Nature (London) 415, 39 (2002).

[4] M. Bartenstein, A. Altmeyer, S. Riedl, S. Jochim, C. Chin, J. H. Denschlag, and R. Grimm, Crossover from a Molecular Bose-Einstein Condensate to a Degenerate Fermi Gas, Phys. Rev. Lett. 92, 120401 (2004).

[5] C. A. Regal, M. Greiner, and D. S. Jin, Observation of Resonance Condensation of Fermionic Atom Pairs, Phys. Rev. Lett. 92, 040403 (2004).

[6] M. W. Zwierlein, C. A. Stan, C. H. Schunck, S. M. F. Raupach, A. J. Kerman, and W. Ketterle, Condensation of Pairs of Fermionic Atoms near a Feshbach Resonance, Phys. Rev. Lett. 92, 120403 (2004).
[7] T. Bourdel, L. Khaykovich, J. Cubizolles, J. Zhang, F. Chevy, M. Teichmann, L. Tarruell, S. J. J. M. F. Kokkelmans, and C. Salomon, Experimental Study of the BEC-BCS Crossover Region in Lithium 6, Phys. Rev. Lett. 93, 050401 (2004).

[8] F. Schreck, L. Khaykovich, K. L. Corwin, G. Ferrari, T. Bourdel, J. Cubizolles, and C. Salomon, Quasipure BoseEinstein Condensate Immersed in a Fermi Sea, Phys. Rev. Lett. 87, 080403 (2001).

[9] A. G. Truscott, K. E. Strecker, I. W. McAlexander, G. B. Partridge, and R. G. Hulet, Observation of Fermi pressure in a gas of trapped atoms, Science 291, 2570 (2001).

[10] I. Ferrier-Barbut, M. Delehaye, S. Laurent, A. T. Grier, M. Pierce, B. S. Rem, F. Chevy, and C. Salomon, A mixture of Bose and Fermi superfluids, Science 345, 1035 (2014).

[11] Z. Hadzibabic, C. A. Stan, K. Dieckmann, S. Gupta, M. W. Zwierlein, A. Görlitz, and W. Ketterle, Two-Species Mixture of Quantum Degenerate Bose and Fermi Gases, Phys. Rev. Lett. 88, 160401 (2002).

[12] K. Günter, T. Stöferle, H. Moritz, M. Köhl, and T. Esslinger, Bose-Fermi Mixtures in a Three-Dimensional Optical Lattice, Phys. Rev. Lett. 96, 180402 (2006). 
[13] E. Wille, F. M. Spiegelhalder, G. Kerner, D. Naik, A. Trenkwalder, G. Hendl, F. Schreck, R. Grimm, T. G. Tiecke, J. T. M. Walraven, S. J. J. M. F. Kokkelmans, E. Tiesinga, and P. S. Julienne, Exploring an Ultracold Fermi-Fermi Mixture: Interspecies Feshbach Resonances and Scattering Properties of ${ }^{6} \mathrm{Li}$ and ${ }^{40} \mathrm{~K}$, Phys. Rev. Lett. 100, 053201 (2008).

[14] A.-C. Voigt, M. Taglieber, L. Costa, T. Aoki, W. Wieser, T. W. Hänsch, and K. Dieckmann, Ultracold Heteronuclear FermiFermi Molecules, Phys. Rev. Lett. 102, 020405 (2009).

[15] A. Trenkwalder, C. Kohstall, M. Zaccanti, D. Naik, A. I. Sidorov, F. Schreck, and R. Grimm, Hydrodynamic Expansion of a Strongly Interacting Fermi-Fermi Mixture, Phys. Rev. Lett. 106, 115304 (2011).

[16] M. Jag, M. Zaccanti, M. Cetina, R. S. Lous, F. Schreck, R. Grimm, D. S. Petrov, and J. Levinsen, Observation of a Strong Atom-Dimer Attraction in a Mass-Imbalanced Fermi-Fermi Mixture, Phys. Rev. Lett. 112, 075302 (2014).

[17] D. Jaksch and P. Zoller, The cold atom Hubbard toolbox, Ann. Phys. 315, 52 (2005).

[18] Y. Kato, K. A. Al-Hassanieh, A. E. Feiguin, E. Timmermans, and C. D. Batista, Novel polaron state for single impurity in a bosonic Mott insulator, Europhys. Lett. 98, 46003 (2012).

[19] F. M. Cucchietti and E. Timmermans, Strong-Coupling Polarons in Dilute Gas Bose-Einstein Condensates, Phys. Rev. Lett. 96, 210401 (2006).

[20] D. Blume, Few-body physics with ultracold atomic and molecular systems in traps, Rep. Prog. Phys. 75, 046401 (2012).

[21] E. Zohar, J. I. Cirac, and B. Reznik, Quantum simulations of lattice gauge theories using ultracold atoms in optical lattices, Rep. Prog. Phys. 79, 014401 (2016).

[22] J. Zhang, J. Unmuth-Yockey, J. Zeiher, A. Bazavov, S.-W. Tsai, and Y. Meurice, Quantum Simulation of the Universal Features of the Polyakov Loop, Phys. Rev. Lett. 121, 223201 (2018).

[23] L. J. LeBlanc and J. H. Thywissen, Species-specific optical lattices, Phys. Rev. A 75, 053612 (2007).

[24] J. Catani, G. Barontini, G. Lamporesi, F. Rabatti, G. Thalhammer, F. Minardi, S. Stringari, and M. Inguscio, Entropy Exchange in a Mixture of Ultracold Atoms, Phys. Rev. Lett. 103, 140401 (2009).

[25] G. Lamporesi, J. Catani, G. Barontini, Y. Nishida, M. Inguscio, and F. Minardi, Scattering in Mixed Dimensions with Ultracold Gases, Phys. Rev. Lett. 104, 153202 (2010).

[26] E. Haller, M. J. Mark, R. Hart, J. G. Danzl, L. Reichsöllner, V. Melezhik, P. Schmelcher, and H.-C. Nägerl, ConfinementInduced Resonances in Low-Dimensional Quantum Systems, Phys. Rev. Lett. 104, 153203 (2010).

[27] F. Schäfer, N. Mizukami, P. Yu, S. Koibuchi, A. Bouscal, and Y. Takahashi, Experimental realization of ultracold $\mathrm{Yb}-{ }^{7} \mathrm{Li}$ mixtures in mixed dimensions, Phys. Rev. A 98, 051602(R) (2018).

[28] H. Ikegami, K. Kim, D. Sato, K. Kono, H. Choi, and Y. P. Monarkha, Anomalous Quasiparticle Reflection from the Surface of a ${ }^{3} \mathrm{He}-{ }^{4} \mathrm{He}$ Dilute Solution, Phys. Rev. Lett. 119, 195302 (2017).

[29] Y. Nishida and S. Tan, Universal Fermi Gases in Mixed Dimensions, Phys. Rev. Lett. 101, 170401 (2008).
[30] Y. Nishida and S. Tan, Confinement-induced $p$-wave resonances from $s$-wave interactions, Phys. Rev. A 82, 062713 (2010).

[31] M. Iskin and A. L. Subaş1, Cooper pairing and BCS-BEC evolution in mixed-dimensional Fermi gases, Phys. Rev. A 82 063628 (2010).

[32] W.-M. Huang, K. Irwin, and S.-W. Tsai, Possible quantum phase manipulation of a two-leg ladder in mixed-dimensional fermionic cold atoms, Phys. Rev. A 87, 031603(R) (2013).

[33] J. Okamoto, L. Mathey, and W.-M. Huang, Fermion pairing in mixed-dimensional atomic mixtures, Phys. Rev. A 95, 053633 (2017).

[34] M. A. Caracanhas, F. Schreck, and C. M. Smith, Fermi-Bose mixture in mixed dimensions, New J. Phys. 19, 115011 (2017).

[35] S. Kelly and S.-W. Tsai, Weak three-dimensional mediators of two-dimensional triplet pairing, Phys. Rev. B 97, 024503 (2018).

[36] Z. Wu and G. M. Bruun, Topological Superfluid in a FermiBose Mixture with a High Critical Temperature, Phys. Rev. Lett. 117, 245302 (2016).

[37] J. M. Midtgaard, Z. Wu, and G. M. Bruun, Topological superfluidity of lattice fermions inside a Bose-Einstein condensate, Phys. Rev. A 94, 063631 (2016).

[38] M. A. Ruderman and C. Kittel, Indirect exchange coupling of nuclear magnetic moments by conduction electrons, Phys. Rev. 96, 99 (1954).

[39] T. Kasuya, A theory of metallic ferro- and antiferromagnetism on zener's model, Prog. Theor. Phys. 16, 45 (1956).

[40] K. Yosida, Magnetic properties of Cu-Mn alloys, Phys. Rev. 106, 893 (1957).

[41] R. Shankar, Renormalization-group approach to interacting fermions, Rev. Mod. Phys. 66, 129 (1994).

[42] C. J. Halboth and W. Metzner, Renormalization-group analysis of the two-dimensional Hubbard model, Phys. Rev. B 61, 7364 (2000).

[43] K.-M. Tam, S.-W. Tsai, and D. K. Campbell, Functional Renormalization Group Analysis of the Half-Filled One-Dimensional Extended Hubbard Model, Phys. Rev. Lett. 96, 036408 (2006).

[44] K.-M. Tam, S.-W. Tsai, and D. K. Campbell, Dominant superconducting fluctuations in the one-dimensional extended Holstein-extended Hubbard model, Phys. Rev. B 89, 014513 (2014).

[45] P. Kopietz, L. Bartosch, and F. Schütz, Introduction to the Functional Renormalization Group (Springer, Berlin, 2010).

[46] W. Metzner, M. Salmhofer, C. Honerkamp, V. Meden, and K. Schönhammer, Functional renormalization group approach to correlated fermion systems, Rev. Mod. Phys. 84, 299 (2012).

[47] C. Platt, W. Hanke, and R. Thomale, Functional renormalization group for multi-orbital Fermi surface instabilities, Adv. Phys. 62, 453 (2013).

[48] J. Voit, One-dimensional Fermi liquids, Rep. Prog. Phys. 58, 977 (1995).

[49] M. Ménard and C. Bourbonnais, Renormalization group analysis of the one-dimensional extended Hubbard model, Phys. Rev. B 83, 075111 (2011).

[50] M. Nakamura, Tricritical behavior in the extended Hubbard chains, Phys. Rev. B 61, 16377 (2000). 
[51] P. Sengupta, A. W. Sandvik, and D. K. Campbell, Bondorder-wave phase and quantum phase transitions in the onedimensional extended Hubbard model, Phys. Rev. B 65, 155113 (2002).

[52] M. Tsuchiizu and A. Furusaki, Phase Diagram of the OneDimensional Extended Hubbard Model at Half Filling, Phys. Rev. Lett. 88, 056402 (2002).
[53] M. Tsuchiizu and A. Furusaki, Ground-state phase diagram of the one-dimensional half-filled extended Hubbard model, Phys. Rev. B 69, 035103 (2004).

[54] A. W. Sandvik, L. Balents, and D. K. Campbell, Ground State Phases of the Half-Filled One-Dimensional Extended Hubbard Model, Phys. Rev. Lett. 92, 236401 (2004). 